\title{
Three solutions for a class of quasilinear elliptic systems involving the $p(x)$-Laplace operator
}

Honghui Yin ${ }^{1,2^{*}}$ and Zuodong Yang ${ }^{1,3}$

\footnotetext{
* Correspondence: yinhh@hytc.edu. cn

'Institute of Mathematics, School of Mathematical Sciences, Nanjing Normal University, Jiangsu Nanjing 210046, China

Full list of author information is available at the end of the article
}

\section{Abstract}

The existence of at least three weak solutions is established for a class of quasilinear elliptic systems involving the $p(x)$-Laplace operator with Neumann boundary condition. The technical approach is mainly based on a three critical points theorem due to Ricceri. MSC: 35D05; 35J60; 58E05.

Keywords: $p(x)$-Laplacian, Sobolev space, three critical points theorem

\section{Introduction}

In this article, we consider the problem of the type

$$
\left\{\begin{array}{l}
-\Delta_{p(x)} u+e_{p}(x)|u|^{p(x)-2} u=\lambda F_{u}(x, u, v)+\mu G_{u}(x, u, v), \quad x \in \Omega, \\
-\Delta_{q(x)} v+e_{p}(x)|v|^{q(x)-2} v=\lambda F_{v}(x, u, v)+\mu G_{v}(x, u, v), \quad x \in \Omega, \\
\frac{\partial u}{\partial v}=\frac{\partial v}{\partial v}=0 \quad x \in \partial \Omega
\end{array}\right.
$$

where $\Omega \subset \mathbf{R}^{N}(N \geq 2)$ is a bounded domain with boundary of class $C^{1} . v$ is the outer unit normal to $\partial \Omega, \lambda, \mu \geq 0$ are real numbers. $p(x), q(x) \in C^{0}(\bar{\Omega})$ with $N<p^{-}:=\inf _{x \in \bar{\Omega}} p(x) \leq p^{+}:=\sup _{x \in \bar{\Omega}} p(x), N<q^{-} \leq q^{+}, F: \Omega \times R \times R \rightarrow R$ is a function such that $F(\cdot, s, t)$ is measurable in $\Omega$ for all $(s, t) \in R \times R$ and $F(x, \cdot, \cdot)$ is $C^{1}$ in $R \times R$ for a.e. $x \in \Omega, F_{s}$ denotes the partial derivative of $F$ with respect to $s$. We assume $G(x$, $s, t)$ and $e_{p}(x), e_{q}(x)$ satisfy the following conditions:

(G) $G: \Omega \times R \times R \rightarrow R$ is a Carathéodory function, $\sup _{\{|s| \leq \theta,|t| \leq 9\}}|G(\cdot, s, t)| \in L^{1}(\Omega)$ for all $\theta, \vartheta>0$;

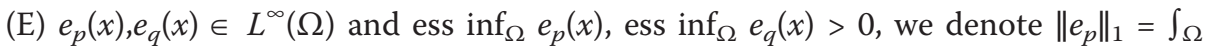
$e_{p}(x) d x$ and $\left\|e_{q}\right\|_{1}=\int_{\Omega} e_{q}(x) d x$.

It is well known that the operator $-\Delta_{p(x)}=-\operatorname{div}\left(|\nabla u|^{p(x)-2} \nabla u\right)$ is called $p(x)$-Laplacian and the corresponding problem is called a variable exponent elliptic systems. The study of differential equations and variational problems with nonstandard $p(x)$-growth conditions has been attracting attention of many authors in the last two decades. It arises from nonlinear elasticity theory, electro-rheological fluids, etc. see [1,2], many results have been obtained on this kind of problems, for example [3-9]. For the special case, $p(x) \equiv p$ (a constant), (1.1) becomes the well known $p$-Laplacian problem. There have been many papers on this class of problems, see [10-19] and the reference therein.

(C) 2012 Yin and Yang; licensee Springer. This is an Open Access article distributed under the terms of the Creative Commons Attribution License (http://creativecommons.org/licenses/by/2.0), which permits unrestricted use, distribution, and reproduction in any medium, provided the original work is properly cited. 
Recently, many papers have appeared in which the technical approach adopted is based on the three critical points theorem obtained by Ricceri [16]. We cite papers [20-23], where the authors established the existence of at least three weak solutions to the problems with Dirichlet or Neumann boundary value conditions. Li and Tang in [24] obtained the existence of at least three weak solutions to problem (1) when $p(x) \equiv$ $p$ with Dirichlet boundary value conditions. El Manouni and Kbiri Alaoui [25] obtained the existence of at least three solutions of system (1) when $p(x) \equiv p$ in $\Omega$ by the three critical points theorem obtained by Ricceri [26].

The main purpose of the present paper is to prove the existence of at least three solutions of problem (1). We study problem (1) by using the three critical points theorem by Ricceri [26] too. On the basis of [27], we state an equivalent formulation of the three critical points theorem in [26] as follows.

Theorem 1. Let $X$ be a reflexive real Banach space, $\Phi: X \rightarrow R$ a continuously Gâteaux differentiable and sequentially weakly lower semicontinuous $C^{1}$ functional, bounded on each bounded subset of $X$, whose Gâteaux derivative admits a continuous inverse on $X^{*} ; \Psi: X \rightarrow R$ a $C^{1}$ functional with compact Gâteaux derivative. Assume that

(i) $\lim _{\|u\| \rightarrow \infty}(\Phi(u)+\lambda \Psi(u))=\infty$ for all $\lambda>0$; and there are $r \in R$ and $u_{0}, u_{1} \in X$ such that:

(ii) $\Phi\left(u_{0}\right)<r<\Phi\left(u_{1}\right)$;

(iii) $\inf _{u \in \Phi^{-1}((-\infty, r])} \Psi(u)>\frac{\left(\Phi\left(u_{1}\right)-r\right) \Psi\left(u_{0}\right)+\left(r-\Phi\left(u_{0}\right)\right) \Psi\left(u_{1}\right)}{\Phi\left(u_{1}\right)-\Phi\left(u_{0}\right)}$.

Then there exists a non-empty open set $\Lambda \subseteq[0, \infty)$ and a positive real number $\rho$ with the following property: for each $\lambda \in \Lambda$ and every $C^{1}$ functional $J: X \rightarrow R$ with compact Gâteaux derivative, there exists $\sigma>0$ such that for each $\mu \in[0, \sigma]$, the equation

$$
\Phi^{\prime}(u)+\lambda \Psi^{\prime}(u)+\mu J^{\prime}(u)=0
$$

has at least three solutions in $X$ whose norms are less than $\rho$.

The paper is organized as follows. In section 2, we recall some facts that will be needed in the paper. In section 3, we establish our main result.

\section{Notations and preliminaries}

In order to deal with $p(x)$-Laplacian problem, we need some theories on spaces $L^{p(x)}$ $(\Omega), W^{1, p(x)}(\Omega)$ and properties of $p(x)$-Laplacian which we will use later (see $[1,5,28,29])$.

We denote

$$
L^{p(x)}(\Omega)=\left\{u \mid u \text { is a measurable real - valued function on } \Omega, \int_{\Omega}|u(x)|^{p(x)} d x<\infty\right\} .
$$

We can introduce a norm on $L^{p(x)}(\Omega)$ by

$$
|u|_{p(x)}=\inf \left\{\lambda>\left.0\left|\int_{\Omega}\right| \frac{u(x)}{\lambda}\right|^{p(x)} d x \leq 1\right\} .
$$


and $\left(L^{p(x)}(\Omega),|\cdot|_{p(x)}\right)$ becomes a Banach space, and we call it variable exponent Lebesgue space.

The space $W^{1, p(x)}(\Omega)$ is defined by

$$
W^{1, p(x)}(\Omega)=\left\{u \in L^{p(x)}(\Omega)|| \nabla u \mid \in L^{p(x)}(\Omega)\right\},
$$

and it can be equipped with the norm

$$
\|u\|_{p(x)}=|u|_{p(x)}+|\nabla u|_{p(x)}, \quad \forall u \in W^{1, p(x)}(\Omega),
$$

and we call it variable exponent Sobolev space. From [5], we know that spaces $L^{p(x)}$ $(\Omega)$ and $W^{1, p(x)}(\Omega)$ are separable, reflexive and uniform convex Banach spaces.

When $e_{p}(x)$ satisfy $(\mathrm{E})$, we define

$$
L_{e_{p}(x)}^{p(x)}(\Omega)=\left\{u \mid u \text { is a measurable real - valued function, } \int_{\Omega} e_{p}(x)|u(x)|^{p(x)} d x<\infty\right\},
$$

with the norm

$$
|u|_{\left(p(x), e_{p}(x)\right)}=\inf \left\{\lambda>\left.0\left|\int_{\Omega} e_{p}(x)\right| \frac{u(x)}{\lambda}\right|^{p(x)} d x \leq 1\right\},
$$

then $L_{e_{p}(x)}^{p(x)}(\Omega)$ is a Banach space. For any $u \in W^{1, p(x)}(\Omega)$, define

$$
\|u\|_{e_{p}}=\inf \left\{\lambda>\left.0\left|\int_{\Omega}\right| \frac{\nabla u(x)}{\lambda}\right|^{p(x)}+e_{p}(x)\left|\frac{u(x)}{\lambda}\right|^{p(x)} d x \leq 1\right\} .
$$

Then it is easy to see that $\|u\|_{e_{p}}$ is a norm on $W^{1, p(x)}(\Omega)$ equivalent to $\|u\|_{p(x)}$. In the following, we will use $\|\cdot\|_{e_{p}}$ to instead of $\|\cdot\|_{p(x)}$ on $W^{1, p(x)}(\Omega)$. Similarly, we use $\|\cdot\|_{e_{p}}$ to instead of $\|\cdot\|_{q(x)}$ on $W^{1, q(x)}(\Omega)$.

Proposition 1. (see $[1,5]$ ) The conjugate space of $L^{p(x)}(\Omega)$ is $L^{p^{0}(x)}(\Omega)$, where $\frac{1}{p(x)}+\frac{1}{p^{0}(x)}=1$. For any $u \in L^{p(x)}(\Omega)$ and $v \in L^{p^{0}(x)}(\Omega)$, we have

$$
\int_{\Omega}|u v| d x \leq\left(\frac{1}{p^{-}}+\frac{1}{\left(p^{0}\right)^{-}}\right)|u|_{p(x)}|v|_{p^{0}(x)} \leq 2|u|_{p(x)}|v|_{p^{0}(x)} .
$$

Proposition 2. (see [1,5])If we denote $\rho(u)=\int_{\Omega}|u|^{p(x)} d x, \forall u \in L^{p(x)}(\Omega)$, then

(i) $|u|_{p(x)}<1(=1 ;>1) \Leftrightarrow \rho(u)<1(=1 ;>1)$;

(ii) $|u|_{p(x)}>1 \Rightarrow|u|_{p(x)}^{p^{-}} \leq \rho(u) \leq|u|_{p(x)}^{p^{+}} ;|u|_{p(x)}<1 \Rightarrow|u|_{p(x)}^{p^{+}} \leq \rho(u) \leq|u|_{p(x)}^{p^{-}}$;

(iii) $|u|_{p(x)} \rightarrow 0(\infty) \Leftrightarrow \rho(u) \rightarrow 0(\infty)$.

From Proposition 2, the following inequalities hold:

$$
\begin{aligned}
& \|u\|_{e_{p}}^{p^{-}} \leq \int_{\Omega}|\nabla u(x)|^{p(x)}+e_{p}(x)|u(x)|^{p(x)} d x \leq\|u\|_{e_{p}}^{p^{+}}, \text {if }\|u\|_{e_{p}} \geq 1 ; \\
& \|u\|_{e_{p}}^{p^{+}} \leq \int_{\Omega}|\nabla u(x)|^{p(x)}+e_{p}(x)|u(x)|^{p(x)} d x \leq\|u\|_{e_{p}}^{p-} \text {, if }\|u\|_{e_{p}} \leq 1 .
\end{aligned}
$$


Proposition 3.If $\Omega \subset \mathbf{R}^{N}$ is a bounded domain, then the imbedding $W^{1, p(x)}(\Omega) \hookrightarrow C^{0}(\bar{\Omega})$ is compact whenever $N<p^{-}$.

Proof. It is well know that $W^{1, p(x)}(\Omega) \hookrightarrow W^{1, p^{-}}(\bar{\Omega})$ is a continuous embedding, and the embedding $W^{1, p-}(\Omega) \hookrightarrow C^{0}(\bar{\Omega})$ is compact when $N<p^{-}$and $\Omega$ is bounded. So we obtain the embedding $W^{1, p(x)}(\Omega) \hookrightarrow C^{0}(\bar{\Omega})$ is compact whenever $N<p$.

From now on, we denote $X$ by $W^{1, p(x)}(\Omega) \times W^{1, q(x)}(\Omega)$ with the norm

$$
\|z\|=\|u\|_{e_{p}}+\|v\|_{e_{p}} \text { for any } z=(u, v) \in X .
$$

Then $X$ is a separable and reflexive Banach spaces. Naturally, we denote $X^{*}$ by the space $\left(W^{1, p(x)}\right)^{*}(\Omega) \times\left(W^{1, q(x)}\right) *(\Omega)$, the dual space of $X$.

From Proposition 3, we know that when $p^{-}, q^{-}>N$, the embedding $x \hookrightarrow C^{0}(\bar{\Omega}) \times C^{0}(\bar{\Omega})$ is compact, there exist a positive constant $c$ such that

$$
\|z\|_{\infty}=\|u\|_{\infty}+\|v\|_{\infty}=\sup _{x \in \bar{\Omega}}|u(x)|+\sup _{x \in \bar{\Omega}}|v(x)| \leq c\|z\| .
$$

\section{Existence of three solutions}

We define $\Phi, \Psi, J: X \rightarrow R$ by

$$
\begin{aligned}
\Phi(z)=\int_{\Omega} & \frac{1}{p(x)}\left(|\nabla u(x)|^{p(x)}+e_{p}(x)|u(x)|^{p(x)}\right) d x \\
& \quad+\int_{\Omega} \frac{1}{q(x)}\left(|\nabla v(x)|^{q(x)}+e_{p}(x)|v(x)|^{q(x)}\right) d x, \\
\Psi(z)= & -\int_{\Omega} F(x, u, v) d x, \\
J(z)= & \int_{\Omega} G(x, u, v) d x .
\end{aligned}
$$

Then for any $(\zeta, \eta) \in X$,

$$
\begin{aligned}
& \left(\Phi^{\prime}(z),(\zeta, \eta)\right)=\int_{\Omega}|\nabla u|^{p(x)-2} \nabla u \nabla \zeta+e_{p}(x)|u|^{p(x)-2} u \zeta d x \\
& +\int_{\Omega}|\nabla v|^{q(x)-2} \nabla v \nabla \eta+e_{q}(x)|v|^{q(x)-2} v \eta d x \quad \forall z \in X, \\
& \left(\Psi^{\prime}(z),(\zeta, \eta)\right)=\int_{\Omega} F_{u}(x, u, v) \zeta d x-\int_{\Omega} F_{v}(x, u, v) \eta d x, \quad \forall z \in X . \\
& \left(J^{\prime}(z),(\zeta, \eta)\right)=\int_{\Omega} G_{u}(x, u, v) \zeta d x-\int_{\Omega} G_{v}(x, u, v) \eta d x, \quad \forall z \in X .
\end{aligned}
$$

We say that $z=(u, v) \in X$ is a weak solution of problem (1) if for any $(\zeta, \eta) \in X$

$$
\left(\Psi^{\prime}(z),(\zeta, \eta)\right)+\lambda\left(\Psi^{\prime}(z),(\zeta, \eta)\right)+\mu\left(J^{\prime}(z),(\zeta, \eta)\right)=0 .
$$

Thus, we deduce that $z \in X$ is a weak solution of (1) if $z$ is a solution of (2). It follows that we can seek for weak solutions of (1) by applying Theorem 1.

We first give the following result. 
Lemma 1. If $\Phi$ is defined in (6), then $\left(\Phi^{\prime}\right)^{-1}: X^{*} \rightarrow X$ exists and it is continuous.

Proof. First, we show that $\Phi^{\prime}$ is uniformly monotone. In fact, for any $\zeta, \eta \in R^{N}$, we have the following inequality (see [30]):

$$
\left(|\zeta|^{p-2} \zeta-|\eta|^{p-2} \eta\right)(\zeta-\eta) \geq \frac{1}{2^{p}}|\zeta-\eta|, p \geq 2 .
$$

Thus, we deduce that

$$
\begin{aligned}
\left(\Phi^{\prime}\left(z_{1}\right)-\Phi^{\prime}\left(z_{2}\right), z_{1}-z_{2}\right) \geq & \min \left\{\frac{1}{2^{p^{+}}} \frac{1}{2^{q^{+}}}\right\}\left(\min \left\{\left\|u_{1}-u_{2}\right\|_{e_{p}}^{p^{+}},\left\|u_{1}-u_{2}\right\|_{e_{p}}^{p^{-}}\right\}\right. \\
& \left.+\min \left\{\left\|v_{1}-v_{2}\right\|_{e_{q}}^{q^{+}},\left\|v_{1}-v_{2}\right\|_{e_{q}}^{q^{-}}\right\}\right),
\end{aligned}
$$

for any $z_{1}=\left(u_{1}, v_{1}\right), z_{2}=\left(u_{2}, v_{2}\right) \in X$, i.e., $\Phi$ ' is uniformly monotone.

From (3), (4), we can see that for any $z \in X$, we have that

$$
\frac{\left(\Phi^{\prime}(z), z\right)}{\|z\|} \geq \frac{\min \left\{\|u\|_{e_{p}}^{p^{+}}, \mid u \|_{e_{p}}^{p^{-}}\right\}+\min \left\{\|v\|_{e_{p}}^{p^{+}},\|v\|_{e_{p}}^{p^{-}}\right\}}{\|u\|_{e_{p}}+\|v\|_{e_{p}}} .
$$

That's meaning $\Phi$ ' is coercive on $X$.

By a standard argument, we know that $\Phi$ ' is hemicontinuous. Therefore, the conclusion follows immediately by applying Theorem 26.A [31].

To obtain our main result, we assume the following conditions on $F(x, s, t)$ :

(A1) There exist $d(x) \in L^{1}(\Omega)$ and $0<\varsigma<p^{-}, 0<\tau<q^{-}$such that

$$
F(x, s, t) \leq d(x)\left(1+|s|^{\zeta}+|t|^{\tau}\right)
$$

for a.e. $x \in \Omega$ and $(s, t) \in R \times R$;

(A2) $F(x, 0,0)=0$ for a.e. $x \in \Omega$;

(A3) There exist $s_{1}, t_{1} \in R$ with $\left|s_{1}\right|,\left|t_{1}\right| \geq 1$ such that

$$
\operatorname{meas}(\Omega) \sup _{(x,|s,| t \mid) \in \Omega \times\left[0, c k_{p}\right] \times\left[0, c k_{q}\right]} F(x, s, t) \leq \frac{\left(\frac{\left\|e_{p}\right\|_{1}}{p^{+}}+\frac{\left\|e_{q}\right\|_{1}}{q^{+}}\right) \int_{\Omega} F\left(x, s_{1}, s_{1}\right) d x}{\frac{\left\|e_{p}\right\|_{1}}{p^{-}}\left|s_{1}\right|^{p^{+}}+\frac{\left\|e_{q}\right\|_{1}}{q^{-}}\left|t_{1}\right|^{q^{+}}},
$$

where $c$ is given in (5) and

$$
\begin{aligned}
& k_{p}=\max \left\{\left(\left\|e_{p}\right\|_{1}+\frac{p^{+}\left\|e_{q}\right\|_{1}}{q^{+}}\right)^{\frac{1}{p^{+}}},\left(\left\|e_{p}\right\|_{1}+\frac{p^{+}\left\|e_{q}\right\|_{1}}{q^{+}}\right)^{\frac{1}{p^{-}}}\right\}, \\
& k_{q}=\max \left\{\left(\frac{q^{+}\left\|e_{p}\right\|_{1}}{p^{+}}+\left\|e_{q}\right\|_{1}\right)^{\frac{1}{q^{+}}},\left(\frac{q^{+}\left\|e_{p}\right\|_{1}}{p^{+}}+\left\|e_{q}\right\|_{1}\right)^{\frac{1}{q^{-}}}\right\} .
\end{aligned}
$$

(A3)' $F(x, s, t)>0$ for any $x \in \Omega$ and $|s|$ or $|t|$ large enough, and there exist $M, N>0$ such that

$$
F(x, s, t) \leq 0, x \in \Omega,|s| \leq M,|t| \leq N
$$

Then we have the following main theorem. 
Theorem 2. Assume (A1),(A2),(A3)(or $\left.(A 3)^{\prime}\right),(G)$ and $(E)$ hold. Then there exist an open interval $\Lambda \subseteq[0, \infty)$ and a positive real number $\rho$ with the following property: for each $\lambda \in \Lambda$, there exists $\sigma>0$ such that for each $\mu \in[0, \sigma]$, problem (1) has at least three weak solutions whose norms are less than $\rho$.

Proof. By the definitions of $\Phi, \Psi$, J, we know that $\Psi$ ' is compact, $\Phi$ is weakly lower semi-continuous and bounded on each bounded subset of $X$. From lemma 1 we can see that $\left(\Phi^{\prime}\right)^{-1}$ is well defined, from condition $(G), J$ is well defined and continuously Gâteaux differentiable on $X$, with compact derivative. Then we can use Theorem 1 to obtain the result. Now we show that the hypotheses of Theorem 1 are fulfilled.

Thanks to (A1), for each $\lambda \geq 0$, one has that

$$
\lim _{\|z\| \rightarrow \infty} \Phi(z)+\lambda \Psi(z)=+\infty,
$$

and so the assumption (i) of Theorem 1 holds.

Now we consider in two cases:

Case (i): (A3) holds, i.e., there exist $1 \leq\left|s_{1}\right|,\left|t_{1}\right|$ such that (9) hold.

Now we set $z_{0}=(0,0), z_{1}=\left(s_{1}, s_{1}\right)$ and denote $r=\frac{\left\|e_{p}\right\|_{1}}{p^{+}}+\frac{\left\|e_{q}\right\|_{1}}{q^{+}}>0$, then it is easy to see that

$$
\Phi\left(z_{1}\right)>r>0=\Phi\left(z_{0}\right) .
$$

Thus, (ii) of Theorem 1 is satisfied.

At last, by (A2) we know $\Psi\left(z_{0}\right)=0$, then

$$
\begin{aligned}
& \frac{\left(\Phi\left(z_{1}\right)-r\right) \Psi\left(z_{0}\right)+\left(r-\Phi\left(z_{0}\right)\right) \Psi\left(z_{1}\right)}{\Phi\left(z_{1}\right)-\Phi\left(z_{0}\right)} \\
& =r \frac{\Psi\left(z_{1}\right)}{\Phi\left(z_{1}\right)} \leq-r \frac{\int_{\Omega} F\left(x, s_{1}, s_{1}\right) d x}{\frac{\left|s_{1}\right|^{+}}{p^{-}}\left\|e_{p}\right\|_{1}+\frac{\mid t_{1} q^{q^{+}}}{q^{-}}\left\|e_{q}\right\|_{1}} .
\end{aligned}
$$

On the other way, when $\Phi(z) \leq r$, we have

$$
\min \left\{\|u\|_{e_{p}}^{p^{+}},\|u\|_{e_{p}}^{p^{-}}\right\} \leq r p^{+}, \min \left\{\|v\|_{e_{q}}^{p^{+}},\|v\|_{e_{q}}^{p^{-}}\right\} \leq r q^{+} .
$$

We deduce that

$$
\|u\|_{e_{p}} \leq \max \left\{\left(r p^{+}\right)^{\frac{1}{p^{+}}},\left(r p^{+}\right)^{\frac{1}{p^{-}}}\right\}
$$

and

$$
\|v\|_{e_{q}} \leq \max \left\{\left(r q^{+}\right)^{\frac{1}{q^{+}}},\left(r q^{+}\right)^{\frac{1}{q^{-}}}\right\}
$$

For $r=\frac{\left\|e_{p}\right\|_{1}}{p^{+}}+\frac{\left\|e_{q}\right\|_{1}}{q^{+}}$, then we have

$$
\|u\|_{e_{p}} \leq k_{p},\|v\|_{e_{q}} \leq k_{q} .
$$

By (5), we obtain

$$
\|u\|_{\infty} \leq c k_{p},\|v\|_{\infty} \leq c k_{q} .
$$


Thus, from (7), we have

$$
\begin{aligned}
-\inf _{z \in \Phi^{-1}((-\infty, r])} \Psi(z) & =\sup _{z \in \Phi^{-1}((-\infty, r])}-\Psi(z) \\
& \leq \int_{\Omega} \sup _{(|u|,|v|) \in\left[0, c k_{p}\right] \times\left[0, c k_{q}\right]} F(x, u, v) d x \\
& \leq \operatorname{meas}(\Omega) \sup _{(x,|u|,|v|) \in \Omega \times\left[0, c k_{p}\right] \times\left[0, c k_{q}\right]} F(x, u, v)
\end{aligned}
$$

From (9)-(11) and the definition of $r$, we can see (iii) of Theorem 1 is hold.

Case (ii): (A3)' holds. Then there exist $\left|s_{2}\right|,\left|t_{2}\right|>1$ such that $F\left(x, s_{2}, t_{2}\right)>0$ for any $x \in$ $\Omega$ and $\left|s_{2}\right|^{p^{-}}\left\|e_{p}\right\|_{1} \geq 1,\left|t_{2}\right|^{q^{-}}\left\|e_{q}\right\|_{1} \geq 1$. Set $a=\min \{c, M\}, b=\min \{c, N\}$ then we have

$$
\int_{\Omega} \sup _{(|s|,|t|) \in[0, a] \times[0, b]} F(x, s, t) d x \leq 0<\int_{\Omega} F\left(x, s_{2}, t_{2}\right) d x .
$$

We denote $z_{2}=\left(s_{2}, t_{2}\right)$ and $r=\min \left\{\frac{1}{p^{+}}\left(\frac{a}{c}\right)^{p^{+}}, \frac{1}{q^{+}}\left(\frac{b}{c}\right)^{q^{+}}\right\}$. Then it is easy to see that

$$
\Phi\left(z_{2}\right)>r>\Phi\left(z_{0}\right) .
$$

So, (ii) of Theorem 1 is satisfied.

When $\Phi(z) \leq r$, similar to the above arguments, we obtain that

$$
\|u\|_{\infty} \leq a,\|v\|_{\infty} \leq b .
$$

At last, we see that

$$
\begin{aligned}
& \frac{\left(\Phi\left(z_{2}\right)-r\right) \Psi\left(z_{0}\right)+\left(r-\Phi\left(z_{0}\right)\right) \Psi\left(z_{2}\right)}{\Phi\left(z_{2}\right)-\Phi\left(z_{0}\right)} \\
= & r \frac{\Psi\left(z_{2}\right)}{\Phi\left(z_{2}\right)} \leq-r \frac{\int_{\Omega} F\left(x, s_{2}, t_{2}\right) d x}{\frac{\left|s_{2}\right|^{+}}{p^{-}}\left\|e_{p}\right\|_{1}+\frac{\left|t_{2}\right|^{+}}{q^{-}}\left\|e_{q}\right\|_{1}}<0 .
\end{aligned}
$$

From (7) and (12), we have

$$
\begin{aligned}
-\inf _{z \in \Phi^{-1}((-\infty, r])} \Psi(z) & =\sup _{z \in \Phi^{-1}((-\infty, r])}-\Psi(z) \\
& \leq \int_{\Omega} \sup _{(|u|,|v|) \in[0, a] \times[0, b]} F(x, u, v) d x \leq 0 .
\end{aligned}
$$

From (14) and (15), we can see (iii) of Theorem 1 is still hold.

Then all the hypotheses of Theorem 1 are fulfilled. By Theorem 1, we know that there exist an open interval $\Lambda \subseteq[0, \infty)$ and a positive constant $\rho$ such that for any $\lambda \in$ $\Lambda$, there exists $\sigma>0$ and for each $\mu \in[0, \sigma]$, problem (1) has at least three weak solutions whose norms are less than $\rho$.

By Theorem 2, we have the following result.

Corollary 1. Let $f, g: \Omega \times R \rightarrow R$ be Carathéodory functions, $\sup _{|\zeta| \leq s}|g(\cdot, \zeta)| \in L^{1}(\Omega)$ for all $s>0$, and define $F(x, t)=\int_{0}^{t} f(x, y) d y$ for any $(x, t) \in \Omega \times R, e(x) \in L^{\infty}(\Omega)$ and ess $\inf _{\Omega} e(x)>0$. Assume the following conditions hold. 
(B1) There exist $d(x) \in L^{1}(\Omega)$ and $0<\varsigma<p^{-}$such that

$$
F(x, t) \leq d(x)\left(1+|t|^{\varsigma}\right)
$$

for a.e.x $\in$ and $t \in R$;

(B2) There exists $t_{3} \in R$ with $\left|t_{3}\right| \geq 1$ such that

$$
\operatorname{meas}(\Omega) \sup _{(x,|s|) \in \Omega \times[0, c k]} F(x, s) \leq \frac{p^{-}}{p^{+}} \frac{\int_{\Omega} F\left(x, t_{3}\right) d x}{\left|t_{3}\right|^{p^{+}}},
$$

where $c$ is given in (5) and

$$
k=\max \left\{\left(\|e\|_{1}\right)^{\frac{1}{p^{+}}},\left(\|e\|_{1}\right)^{\frac{1}{p^{-}}}\right\} ;
$$

or

(B2)' $F(x, t)>0$ for any $x \in \Omega$ and $|t|$ large enough, and there exist $M>0$ such that

$$
F(x, t) \leq 0, x \in \Omega,|t| \leq M .
$$

Then there exist an open interval $\Lambda \subseteq[0, \infty)$ and a positive constant $\rho$ such that for any $\lambda \in \Lambda$, there exists $\sigma>0$ and for each $\mu \in[0, \sigma]$, the problem

$$
\left\{\begin{array}{l}
-\Delta_{p(x)} u+e(x)|u|^{p(x)-2} u=\lambda f(x, u)+\mu g(x, u), \quad x \in \Omega, \\
u=0 \quad x \in \partial \Omega
\end{array}\right.
$$

has at least three weak solutions whose norms are less than $\rho$.

Remark 1. if $p(x)=p$ in $\Omega, \mu=0$, problem (17) was considered in [21]. If we take $f$ $(x, t)=|t|^{\gamma(x)-2} t-t$ with $\gamma(x) \in C^{0}(\bar{\Omega})$ satisfies $2<\gamma \leq \gamma^{+}<p^{-}, \mu=0$, Corollary 1 becomes a version of Theorem 2 in [23]. Hence our Corollary 1 unifies and generalizes Theorem 2 in [21] and Theorem 2 in [23] and our Theorem 2 generalizes the main results of [21-25] to the system (1).

At last, we give two examples.

Example 1. Let $\Omega=B(0,1)$ be the unit ball of $R^{N}$ with $N \geq 2$, set $p(x)=N+e^{|x|}, q(x)$ $=N+1+\ln \left(1+x^{2}\right), e_{p}(x)=\left(1+x^{2}\right)=e_{q}(x), G(x, u, v)=x^{2}\left(u^{2}+v^{2}\right)$ and

$$
F(x, u, v)=\left\{\begin{array}{l}
e^{x^{2}}\left(e^{u}+u v-1\right), \quad x \in \Omega, u \leq M, v \in R, \\
e^{x^{2}}\left(u e^{M}+u v+\frac{1}{2} u^{2}-M u-(M-1) e^{M}+\frac{1}{2} M^{2}\right), \quad x \in \Omega, u \leq M, v \in R,
\end{array}\right.
$$

where $M$ is a positive constant, i.e., we consider the following problem

$$
\left\{\begin{array}{l}
-\Delta_{p(x)} u+\left(1+x^{2}\right)|u|^{p(x)-2} u=\lambda f(x, u, v)+\mu^{2} x^{2} u, \quad x \in \Omega, \\
-\Delta_{q(x)} v+\left(1+x^{2}\right)|v|^{q(x)-2} v=\lambda u+\mu 2 x^{2} v, \quad x \in \Omega, \\
\frac{\partial u}{\partial v}=\frac{\partial v}{\partial v}=0 \quad x \in \partial \Omega .
\end{array}\right.
$$

where

$$
f(x, u, v)=F_{u}(x, u, v)=\left\{\begin{array}{l}
e^{x^{2}}\left(e^{u}+v\right), \quad x \in \Omega, u \leq M, v \in R, \\
e^{x^{2}}\left(e^{M}+v+u-M\right), \quad x \in \Omega, u \leq M, v \in R,
\end{array}\right.
$$

We can see that $p^{+}=N+e, p^{-}=N+1, q^{+}=N+1+\ln 2, q^{-}=N+1,\|e\|_{1}=\frac{4}{3}$, and it is easy to see that for any $t_{1}>1$, there exists $s_{1}>1$ such that 


$$
\frac{e^{s_{1}}+s_{1} t_{1}-1}{\frac{s_{1}^{p^{+}}}{p^{-}}+\frac{t_{1}^{q^{+}}}{q^{-}}} \geq \frac{e\left(e^{c k_{p}}+c^{2} k_{p} k_{q}-1\right)}{\frac{1}{p^{+}}+\frac{1}{q^{+}}}
$$

were $k_{p}=\left(\frac{4}{3}+\frac{4(N+1+\ln +2)}{3(N+e)}\right)^{\frac{1}{N+1}}, k_{q}=\left(\frac{4}{3}+\frac{4(N+e)}{3(N+1+\ln 2)}\right)^{\frac{1}{N+1}}$ are positive constants and $c$ is given by (5). Then when $M \geq s_{1}, F(x, u, v)$ defined in (18) satisfies (A1)(A3) of Theorem 2 , and $G(x, u, v), e(x)$ satisfy

(G) and (E) respectively, by Theorem 2 , there exist an open interval $\Lambda \subseteq[0, \infty)$ and a positive constant $\rho$ such that for any $\lambda \in \Lambda$, there exists $\sigma>0$ and for each $\mu \in[0, \sigma]$, system (19) has at least three weak solutions whose norms are less than $\rho$.

Example 2. Assume $\Omega, p(x), q(x), e_{p}(x), e_{q}(x), G(x, u, v)$ are the same as in example 1, and suppose $N \geq 8$. Let

$$
F(x, u, v)=\left(1+2 x^{2}\right)\left(u^{4} v^{2}+v^{4} u^{2}-2 u^{2} v^{2}\right), \quad x \in \Omega, u, v \in R .
$$

Obviously, $F(x, u, v)$ satisfies (A1) and (A2). By simple computation, we can see that

$$
F(x, u, v)>0, \text { when }|u|>\sqrt{2} \text { or }|v|>\sqrt{2}
$$

and

$$
F(x, u, v)<0 \text {, when }|u|<1 \text { and }|v|<1,
$$

i.e., (A3)' hold for $F(x, u, v)$ defined in (22).

Thus, there exist an open interval $\Lambda \subseteq[0, \infty)$ and a positive constant $\rho$ such that for any $\lambda \in \Lambda$, there exists $\sigma>0$ and for each $\mu \in[0, \sigma]$, the system

$$
\left\{\begin{array}{l}
-\Delta_{p(x)} u+\left(1+x^{2}\right)|u|^{p(x)-2} u=\lambda 4 u^{3} v^{2}+2 v^{4} u-4 u v^{2}+\mu 2 x^{2} u, \quad x \in \Omega, \\
-\Delta_{p(x)} v+\left(1+x^{2}\right)|v|^{q(x)-2} v=\lambda 4 u^{3} v^{2}+2 u^{4} v-4 v u^{2}+\mu 2 x^{2} v, \quad x \in \Omega, \\
\frac{\partial u}{\partial v}=\frac{\partial v}{\partial v}=0 \quad x \in \partial \Omega .
\end{array}\right.
$$

has at least three weak solutions whose norms are less than $\rho$.

Remark 2. We remark that the methods used in this paper are also applicable for the cases of the other boundary value conditions, for example, Dirichlet boundary value conditions.

\section{Acknowledgements}

The project supported by the National Natural Science Foundation of China (No. 11171092). Project supported by the Natural Science Foundation of the Jiangsu Higher Education Institutions of China (Grant No. 08KJB110005).

\section{Author details}

'Institute of Mathematics, School of Mathematical Sciences, Nanjing Normal University, Jiangsu Nanjing 210046, China ${ }^{2}$ School of Mathematical Sciences, Huaiyin Normal University, Jiangsu Huaian 223001, China ${ }^{3}$ College of Zhongbei,

Nanjing Normal University, Jiangsu Nanjing 210046, China

\section{Authors' contributions}

All authors read and approved the final manuscript.

Competing interests

The authors declare that they have no competing interests.

Received: 6 October 2011 Accepted: 7 March 2012 Published: 7 March 2012 


\section{References}

1. Rúzicka, M: Electro-rheological Fluids: Modeling and Mathematical Theory. In Lecture Notes in Math, vol. 1784,SpringerVerlag, Berlin (2000)

2. Zhikov, W: Averaging of functionals of the calculus of variations and elasticity theory. Math USSR IzV. 29, 33-66 (1987). doi:10.1070/IM1987v029n01ABEH000958

3. Acerbi, E, Mingione, G: Regularity results for a class of functionals with nonstandard growth. Arch Ration Mech Anal. 156, 121-140 (2001). doi:10.1007/s002050100117

4. Fan, XL: Solutions for $p(x)$-Laplacian Dirichlet problems with singular coefficients. J Math Anal Appl. 312, 464-477 (2005). doi:10.1016/j.jmaa.2005.03.057

5. Fan, $X L$, Zhao, D: On the spaces $L^{p(x)}(\Omega)$ and $W^{m, p(x)}(\Omega)$. J Math Anal Appl. 263, 424-446 (2001). doi:10.1006/ jmaa.2000.7617

6. Fan, XL, Wu, HQ, Wang, FZ: Hartman-type results for p(t)-Laplacian systems. Nonlinear Anal. 52, 585-594 (2003). doi:10.1016/S0362-546X(02)00124-4

7. El Hamidi, A: Existence results to elliptic systems with nonstandard growth con-ditions. J Math Anal Appl. 300, 30-42 (2004). doi:10.1016/j.jmaa.2004.05.041

8. Zhang, QH: Existence and asymptotic behavior of positive solutions for variable exponent elliptic systems. Nonlinear Anal. 70, 305-316 (2009). doi:10.1016/j.na.2007.12.001

9. Zhang, QH, Qiu, ZM, Dong, R: Existence and asymptotic behavior of positive solutions for a variable exponent elliptic system without variational structure. Nonlinear Anal. 72, 354-363 (2010). doi:10.1016/j.na.2009.06.069

10. Alves, CO, de Morais Filho, DC, Souto, MAS: On systems of elliptic equations involving subcritical or critical Sobolev exponents. Nonlinear Anal. 42, 771-787 (2000). doi:10.1016/50362-546X(99)00121-2

11. Chen, $\mathrm{CH}$ : On positive weak solutions for a class of quasilinear elliptic systems. Nonlinear Anal. 62, 751-756 (2005). doi:10.1016/j.na.2005.04.007

12. Hai, DD, Shivaji, R: An existence result on positive solutions of p-Laplacian systems. Nonlinear Anal. 56, 1007-1010 (2004). doi:10.1016/j.na.2003.10.024

13. Hsu, TS: Multiple positive solutions for a critical quasilinear elliptic system with concave-convex non-linearities. Nonlinear Anal. 71, 2688-2698 (2009). doi:10.1016/..na.2009.01.110

14. Han, P: The effect of the domain topology on the number of positive solutions of elliptic systems involving critical Sobolev exponents. Houston J Math. 32, 1241-1257 (2006)

15. Kristály, A: Existence of two non-trivial solutions for a class of quasilinear elliptic variational systems on strip-like domains. Proc Edinb Math Soc II. 48, 465-477 (2005)

16. Ricceri, B: On a three critical points theorem. Arch Math (Basel). 75, 220-226 (2000). doi:10.1007/s000130050496

17. Wu, TF: The Nehari manifold for a semilinear elliptic system involving sign-changing weight functions. Nonlinear Anal. 68, 1733-1745 (2008). doi:10.1016/j.na.2007.01.004

18. Yin, HH, Yang, ZD: Existence and nonexistence of entire positive solutions for quasilinear systems with singular and super-linear terms. Diff Equ Appl. 2(2):241-249 (2010)

19. Yin, HH, Yang, ZD: Multiplicity results for a class of concave-convex elliptic systems involving sign-changing weight. Ann Pol Math. 102(1):51-71 (2011). doi:10.4064/ap102-1-5

20. Afrouzi, GA, Heidarkhani, S: Three solutions for a Dirichlet boundary value problem involving the $p$-Laplacian. Nonlinear Anal. 66, 2281-2288 (2007). doi:10.1016/j.na.2006.03.019

21. Bonanno, G, Candito, P: Three solutions to a Neumann problem for elliptic equations involving the $p$-Laplacian. Arch Math (Basel). 80, 424-429 (2003)

22. Candito, P: Existence of three solutions for a nonautonomous two point boundary value problem. J Math Anal Appl. 252, 532-537 (2000). doi:10.1006/jmaa.2000.6909

23. Mihăilescu, M: Existence and multiplicity of solutions for a Neumann problem involving the $p(x)$-Laplace operator. Nonlinear Anal. 67, 1419-1425 (2007). doi:10.1016/j.na.2006.07.027

24. Li, C, Tang, CL: Three solutions for a class of quasilinear elliptic systems involving the $(p, q)$-Laplacian. Nonlinear Anal. 69 3322-3329 (2008). doi:10.1016/j.na.2007.09.021

25. El Manouni, S, Kbiri Alaoui, M: A result on elliptic systems with Neumann conditions via Ricceri's three critical points theorem. Nonlinear Anal. 71, 2343-2348 (2009). doi:10.1016/j.na.2009.01.068

26. Ricceri, B: A three critical points theorem revisited. Nonlinear Anal. 70, 3084-3089 (2009). doi:10.1016/j.na.2008.04.010

27. Bonanno, G: A minimax inequality and its applications to ordinary differential equations. J Math Anal Appl. 270 210-229 (2002). doi:10.1016/S0022-247X(02)00068-9

28. Kováčik, O, Rákosník, J: On the spaces $L^{p(x)}(\Omega)$ and $W^{k, p(x)}(\Omega)$. Czechoslovak Math J. 41, 592-618 (1991)

29. Sanko, SG: Denseness of $C_{0}^{\infty}\left(R^{N}\right)$ in the generalized Sobolev spaces $W^{m, p(x)}\left(R^{N}\right)$. Dokl Ross Akad Nauk. 369(4):451-454 (1999)

30. Kichenassamy, S, Veron, L: Singular solutions of the $p$-Laplace equation. Math Ann. 275, 599-615 (1985)

31. Zeider, E: Nonlinear Functional Analysis and its Applications, II/B: Nonlinear Monotone Operators. Springer, New York (1990)

doi:10.1186/1687-2770-2012-30

Cite this article as: Yin and Yang: Three solutions for a class of quasilinear elliptic systems involving the $p(x)$ -

Laplace operator. Boundary Value Problems 2012 2012:30. 